\title{
Motivation for Green Computing, an Analytical Approach
}

\author{
Syed Furqan Qadri, Suneel Shehzad, Muhammad Amjad, Masood Anwar, \\ Muhammad Asif Mahmood Khan, Salman Afsar Awan \\ Department of Computer Science, University of Agriculture Faisalabad 38000, PAKISTAN
}

\begin{abstract}
Depleting wastage in computing tool life cycle from initial launch to delivery green computing is a domain which makes system more energy efficient. The aim of this crucial issue is to introduce eminent research that bespeaks the latest enhancements in the field of green computing. In designing the next generation computing technologies green computing has to be considered as a significant component. In this research different component of green computing are discussed, evolution, applications, thin client, different strategies and its framework. This includes truly sustainable green computing technologies development and related solution. Finally it is demonstrated a framework and a SWOT analysis of green computing.
\end{abstract}

Keywords: Green computing, thin client, green cloud computing framework, SWOT analysis.

\section{Introduction:}

Green computing presented us a novel idea that using technology does not mean to spiflicate our environment to achieve our tasks. Environmental friendly computing can be distinct as practice and examine the manufacturing computer subsystems such as monitors, printers, hard and magnetic disks, networks and communicating devices with proficiency and effectively with no effect and impact on environment [1].

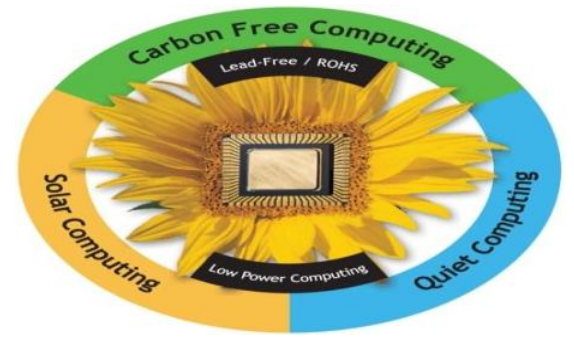

Fig.1

Figure 1 shows how the carbon foot prints of user can be reduce. Different manufacturing companies take their eyes on the sun, and the solar computing opening is a substantial part of its green computing projects. Solar powered devices can use energy efficient processors as they use four times less carbon [7]. Solar cells are cost effective and require little maintenance. It is need of time that Company manufacturing motherboard chipsets, CPUs, and other computer hardware, acquainted its go-ahead for green computing. According to United Kingdom figure that $1 \mathrm{KWh}$ produces 0.1 kilogram of carbon dioxide and $190 \mathrm{kWh}$ creates 1 tons of $\mathrm{co}_{2}$. This figure shows that an office PC costs to operate $£ 16$ annually and generate 1.10 tons of $\mathrm{co}_{2} / \mathrm{yr}^{\mathrm{r}}$. it is anticipated by Bill Gates many years ago that a PC in every home with objective of promoting his company remuneration. Not just in home but also all commercial organizations are dependent on IT field [2]. We utilize little of IT in our home and not leaving our systems switched on unreasonably.

\subsection{Related Work:}

In the recent years it has observed the persisting growing internet from its pilot communicating purpose as email and web where computing and storage potential increased permanently by the end users. According to Gartner report that unprecedented number of PCs is deployed worldwide and its consignment have reached near 83 million units just in second quarter of 2010 indicating a 20.7\% gain from second quarter of 2009 and monumental power have been decreased due to groundless imageries. Like as Dell Core-2 PC consume 85 watts when sitting idle, result to nearly half power when it was fully loaded and same like most idle servers use about 68-97 percent of total energy consumption when they are fully functional [3]. As power costs rising with rise of IT infrastructure, downsizing of running cost is becoming the priority of IT industry.

Francis and Richardson in 2009 introduced a green computing model which main focus was to minimize in power expenditure from system life cycle as a prime motivator for green computing [4]. Some hardware manufacturer researcher from IBM demonstrated a server workload analysis for energy decrease. Basic strategy was putting server on and off [5]. The annual cost of use and wastage by different appliances is given below. 
Table 1: Annual power wastage and cost

\begin{tabular}{|l|c|c|c|c|c|c|}
\hline Appliances & $\begin{array}{c}\text { Hours } \\
\text { per } \\
\text { day }\end{array}$ & $\begin{array}{c}\text { Watts } \\
\text { when } \\
\text { on }\end{array}$ & $\begin{array}{c}\text { Annual } \\
\text { Cost of } \\
\text { use }\end{array}$ & $\begin{array}{c}\text { Watts } \\
\text { on } \\
\text { stand } \\
\text { by }\end{array}$ & $\begin{array}{c}\text { Annual } \\
\text { cost on } \\
\text { standby }\end{array}$ & $\begin{array}{c}\% \\
\text { wastage }\end{array}$ \\
\hline TV & 2 & 75 & $£ 7.12$ & 14 & $£ 14.61$ & $67 \%$ \\
\hline VCR & 0.1 & 15 & $£ 0.07$ & 8 & $£ 9.07$ & $99 \%$ \\
\hline Computer & 2 & 60 & $£ 5.69$ & 13 & $£ 13.57$ & $70 \%$ \\
\hline Microwave & 0.25 & 1700 & $£ 20.17$ & 24 & $£ 27.05$ & $57 \%$ \\
\hline $\begin{array}{l}\text { Battery } \\
\text { Charger }\end{array}$ & 6 & 12 & $£ 3.41$ & 10 & $£ 3.87$ & $53 \%$ \\
\hline
\end{tabular}

A personal computer consists of central processing unit, monitor, printer and other peripheral devices. In system CPU need nearby 200W power and for a 17-19 inch monitor require $70-150 \mathrm{~W}$ power in 8 hours daily use.

- A conventional laser printer utilizes 100 watts of power

- When a user actuates the computer also factor of power expenses

- 12 watts of energy utilizes inkjet printer when printing and 5 watts when idle

- Suppose you operate a PC that consumes $200 \mathrm{~W}$ in day and night every day its annual cost will be above $£ 16$.

- Managing your system for business purpose 40 hours per week then total power cost would be about $\$ 30$.

\section{Evaluation:}

Power consumption can be measure by these two commercially available devices as in figure 2 .
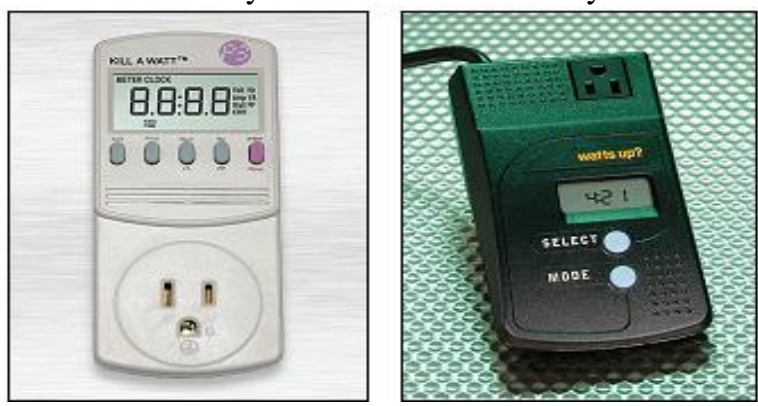

Fig.2 Power utilization measurements devices

20 inch Dell 2005 FPW wide screen LCD monitors was tested in unplugged state which demonstrates the expected zero watt draw. It draws $1 \mathrm{~W}$ when plugged but power off, it drew $3 \mathrm{~W}$ in sleep mode and when monitor is on it drew $55 \mathrm{~W}$. This distinction in power use highlights the power saving that is available. $94 \%$ power is saved when monitor is in sleep mode. Similarly $97 \%$ power is saved when in hibernate modes [8].

Mathematically equation has been formulated to approximation measurement of PCs power usage and for other power consuming devices. Computer or other appliances power consumption dependent on which type of work is performed. For example computer average power utilization in computer gaming is higher than it is being used for browsing. Expenses involved in running a computer can be estimated by applying watts/hour. The cost to run a computer for one year is calculated by using local cost of electricity per $\mathrm{kWh}$ as:

Cost yearly $=$ [avg. watts/hours $]^{*}[\text { hours run in one year }]^{*}[1 \mathrm{kWh} \text { per } 1000 \text { watts }]^{*}[$ cost power $/ \mathrm{kWh}]$

\section{Applicability To Computer}

Role of computer science in the research, teaching and encouragements of green computing is essential. Computer science researchers and educationists are serving in promoting green computing. Some are green computing technologies skill for students and faculty to educate others about this discipline [9]. Few of green commuting strategies in computer science as:

- Power saving modes of computers

- System standby mode

- Sleep mode of monitor

- Apply screen saver

- Sleep mode of hard disk

- Up gradation to extend computer lifecycle 
- Purchase of more efficient computer devices

- Turn off equipment when not in use

- Convert monitor to LCD and LCD to LED technology

- Use AMD processors

3.1 Our contribution in green computing:

- Turning PC's off at night will save energy up to $810 \mathrm{kWh}$ per year and $67 \%$ net annul savings

- Flat screen monitor use less power than CRTs.

- When device is not in use unplug it.

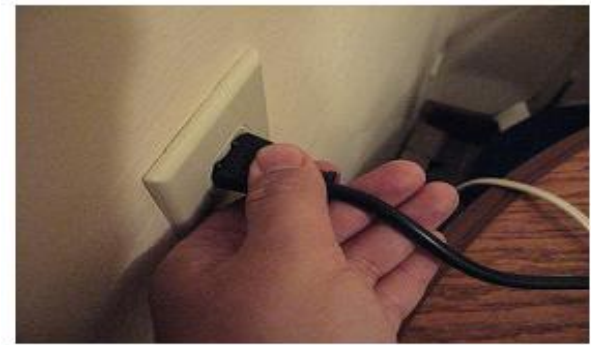

Fig.3 Unplug the device

- 14 inch monitor saves $40 \%$ power than 17 inch monitor.

- Facilitate the sleep/standby and power supervision setting on PCs

- Power off monitor when not in use

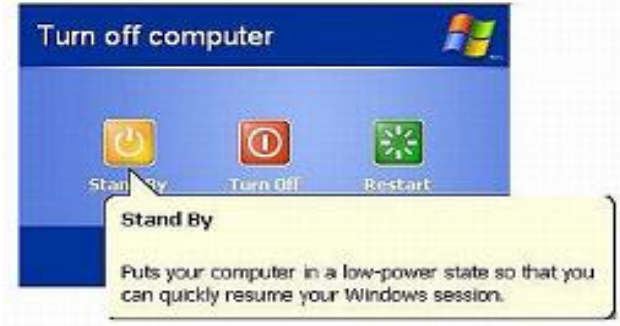

Fig.4 PC standby mode

\subsection{Green computing strategies:}

- Green computing reduce the amount of pollutants in the atmosphere

- It shrinks the paper industry burden

- It saves power consumption and reduce $\mathrm{co}_{2}$ created from computer

- Promote the use of renewable resources

- Promote the alternative energy source as solar system

\section{Thin Clients:}

A scatted computing infrastructure consists of desktop and laptop while many enterprises rely on laptop as each personal computer. Like as thin computer system use lightweight workstation that has not capability of local computation, such systems handle user inputs and simply display graphics. In thin clients, servers are centralizing for the calculation of many end users. The aim of this approach is to show energy potential. Thin clients require little energy and strength workloads take to higher usage on multiplexed servers. Extreme version of green computing is shown by thin client systems.

\section{Green Cloud Computing Framework:}

Increase in expenditure both economical and environmental provokes for the efficient and scalable green cloud computing. In a quickly changing environment it is mandatory to meet these needs both for business and educational institutions. Green cloud computing is basically a specific discipline of green computing that is concern with energy efficient clouds in computing process. In order to meet the goal of reducing power usage a framework of green cloud computing is presented here that is useful to the cloud. To determine efficient computing recourse a framework is designed. Green computing technologies can be customized and functional to cloud computing. 


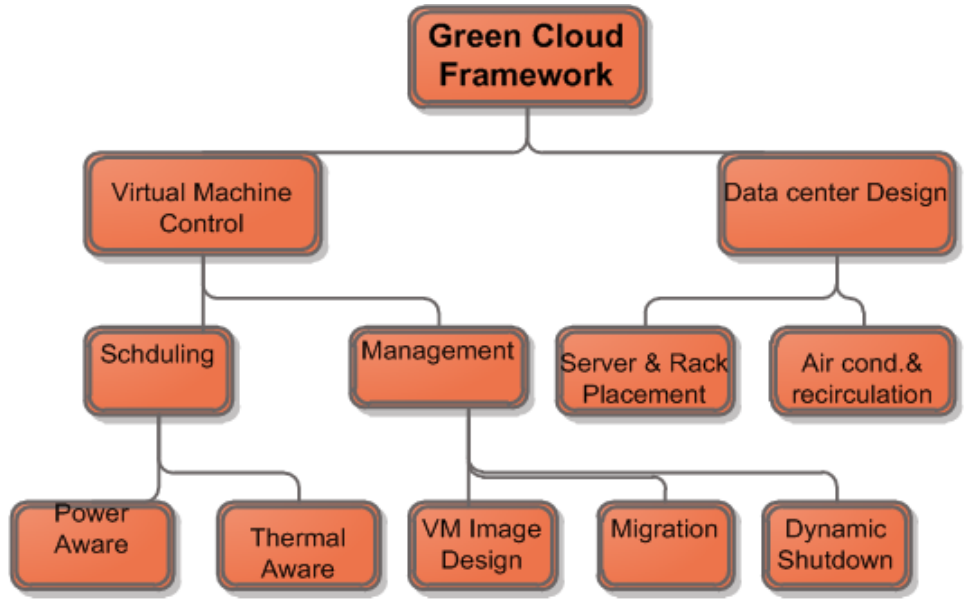

Fig. 5 Green cloud computing framework

Figure 5 shows a green cloud computing framework for exploiting performance per watt with a cloud. VM arrangement, VM image management and advances data center design are major areas of green cloud framework. There are two major regions within framework; here it can be observe 5\% performance failure is due to $18 \%$ attenuation in frequency. This allows for creation of an efficient scheduling system that attempting to maximize performance while minimize power expenditure of the system [11]. The energy performance exchange within cluster is completed by the energy efficient scheduler. Advanced features set of cloud could yield drastic results by combing various power efficiency techniques for data centers. In this context first we have to boost upon baseline function of virtual machine in cloud to find a more efficient training system for VMs. While reducing the operational cost of cloud the scheduling portion itself deals with the assigning of VMs within cloud infrastructure. This is achieved by optimize power of server instrumentation itself or overall temperature of datacenters. To further improve efficiency, we can mold the ability to proceed and handling of VMs because of intrinsically disposability and mobility of VMs within semi homogeneous data center. To preserve power image section handles and influence the size and positioning of VM images in different ways [10] and aim of VMs images is firm power saving.

So it can have far reaching effect due to functional and runtime probability; Decision related to static datacenter design should be incorporated. By applying more efficient power supplies for servers can direct to incremental but substantive improvement by using air conditioning units employing cooling. The integrated components of green cloud computing framework provide a sustainable exploitation stage which is largest potential link factor to significantly energy requirement within cloud data center. Many challenges with large scale cloud are created to combine each factor together in the framework.

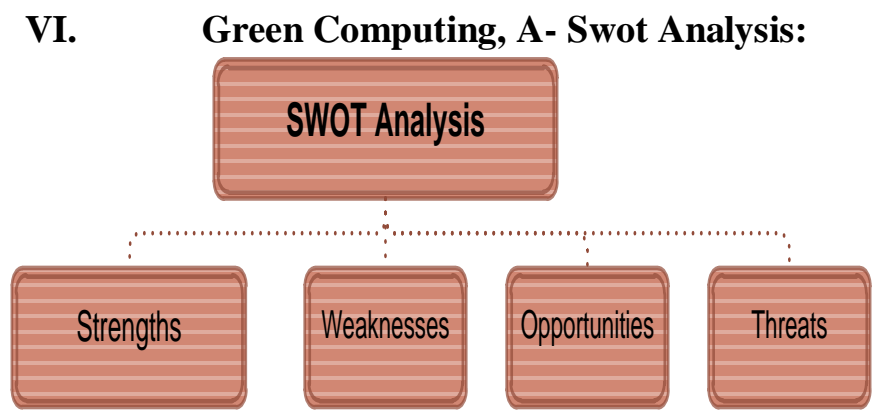

\subsection{Strength:}

Fig. 6 SWOT analysis

Computing technology and its infrastructure plays a vital role in human life and subsequently the associated its high intensity of power expenditure both economically and environmentally is a major concern. Green computing is emerging a prompting solution which is arising computing technology that cut down power usage that leads significantly $\mathrm{CO}_{2}$ emission reduction [6]. Next generation information and communication technology designers have to consider green computing as an essential component. Energy efficient CPUs, servers and peripheral devices as well as proper disposal of electronic wastage are the key points which are advocated by green computing. 


\subsection{Weakness:}

IT architects have given much importance to security because of past consequences. Environment friendly qualities are becoming architectural issues for new projects to reduce power consumption is obvious likewise security. Now a day's new techniques are being promoted as data centers are being sized by watt. Power saving is yet another architectural problem. Techniques like power management, optimizing operational processes and continuously reducing environmental implications are more challenging. There is consensus that negative environment repercussions are the fallout of synthetic pollution [12].

\subsection{Opportunities:}

One of the fastest growing sectors of U.S.A economy is the IT industry in energy consumption point of view [11]. Hybrid computers indicate an opportunity to make novel use of decades of system processor migration research and multi core process placement to reduce pollutant wastage. The aim of operating system is to support hybrid computing would benefit from knowledge of which applications are better suited for local execution and which process could be executed faster from cloud.

\subsection{Threats:}

Main biggest threats to green computing are the possibility of repercussion from trenched incumbents while many forward looking organizations will see green computing as an opportunity to migrate to best computing practices that open up exciting for in house IT staff. There will probability be many IT institutions will view it as a threat to their IT culture in terms of data security and IT audit policies etc [11]. Beside this small business organizations have been quick to adapt and even welcome green computing; large corporate customers have voiced a superfluity of concern about handling their operations to another company.

\section{Conclusion And Future Work:}

Modern computing poses not only technology problems, but it has to facing major environmental challenges in term of its high power consumption. With increase in IT infrastructure, it is clear point that effective green computing policy must be formulated to minimize its environmental effects. To fulfill the user requirements and workload it is advocated to substitute desktops with laptops. We plan to achieve a broader study to enhance the green computing strategies which execute in enormously disciplines and different of its types that circulates computing. Additional guideline improvement is considered which objective of engaging researchers, faculty members and students in green computing as $21^{\text {st }}$ century hottest topic of research in computer sciences and engineering. Through that green computing will gain more public and researcher attention by work of environmental and governmental organizations?

\section{Acknowledgments:}

The authors would like to acknowledge Salman Afsar Awan for the comments provided while preparing this work and for the feedback and suggestions provided on an early version of the article.

\section{References:}

[1] (2006-10-23). "EPA Announces New Computer Efficiency Requirements". A report regulated by Jones, Ernesta.

[2] IBM Global Technology Services January 2007 report.

[3] Natural Resources Defense Council "Recommendations for $\quad$ Tier I I ENERG ComputerSpecification”,,www.energystar.gov/ia/partners/prod_development/revisions/downloads/computer/ RecommendationsTierICompSpecs.pdf.

[4] Kevin Francis, Peter Richardson, Green Maturity Model for Virtualization, http://msdn.microsoft.com/enus/library/dd393310.aspx.

[5] AkshatVerma, GargiDasgupta, Tapan Kumar Nayak, Pradipta De, Ravi Kothari, Server workload analysis for power minimi zation using consolidation. in:Proceedings of the 2009 Conferenceon USENIX Annual Technical Conference, USENIX'09, USENIX Association, Berkeley, CA, USA, 2009, pp.28-28.

[6] R. Zhu, Z. Sun, and J. Hu, "Special section: Green computing," Future Generation Computer Systems, vol. 28, no. 2, pp. 368-370, Feb. 2012.

[7] S. Marston, Z. Li, S. Bandyopadhyay, J. Zhang, and A. Ghalsasi, "Cloud computing — The business perspective," Decision Support Systems, vol. 51, no. 1, pp. 176-189, Apr. 2011.

[8] MujtabaTalebi. Computer Power Consumption Benchmarking For Green Computing.Master's Thesis, Villanova University, Department of Computing Sciences, 2008.

[9] Colin Holland. Oxford University launches low carbon computing project. EE Times UK, March 14, 2008.

[10] Younge, A. J., Laszewski, G. V., Wang, L., Lopez-alarcon, S., \&Carithers, W. (2008). Efficient Resource Management for Cloud Computing Environments, (C), 2005-2008.

[11] C. Lin, "A Novel Green Cloud Computing Framework for Improving System Efficiency," Physics Procedia, vol. 24, pp. 23262333, Jan. 2012.

[12] B. Dougherty, J. White, and D. C. Schmidt, "Model-driven auto-scaling of green cloud computing infrastructure," Future Generation Computer Systems, vol. 28, no. 2, pp. 371-378, 2012. 


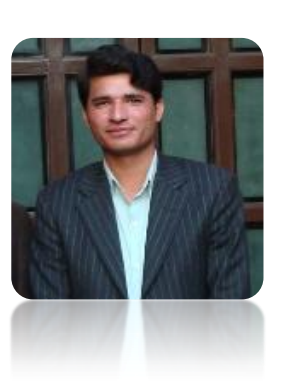

\section{Author's Profile:}

Syed Furqan Qadri received M.Sc (Computer Science) degree in 2011 and MS (CS) degree from University of Agriculture, Faisalabad (UAF) in 2013. His current research interests are in green computing, topological scenarios suitability for wireless body area network (WBAN) using IEEE 802.15.4/zigbee standard, bandwidth allocation, admission control, and fade countermeasure systems in terrestrial wireless and satellite multi-service networks.

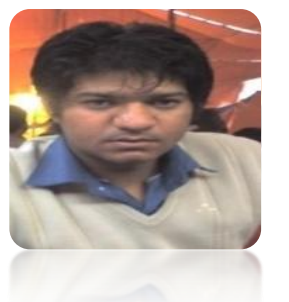

Suneel Shehzad received BS(CS) degree from University of central Punjab in 2009 and MS(CS) from University of Agriculture Faisalabad in 2013. Currently working on the performance analysis of Adhoc Network Protocols, Green Computing and Wireless Body Area Network (WBAN).

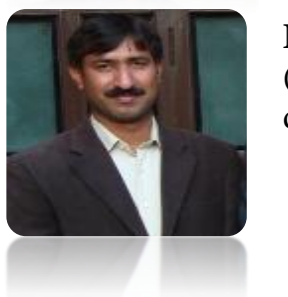

Muhammad Amjad received MCS degree from Hamdard University. Now a student of MS (CS) from University of Agriculture, Faisalabad. His research area is Agile approach in user design interaction, software quality assurance and Green cloud computing.

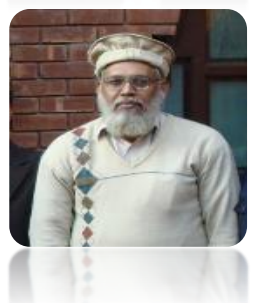

Masood Anwar received MCS degree from Sarhad University Peshawar and MS (CS) from university of Agriculture Faisalabad. He has an experience of 12 years working in Networks and Communication. His research area is security and threats in networks and green computing.

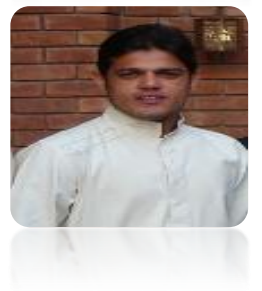

M.Asif Mahmood Khan received BS (CS) from GC University Faisalabad and MS (CS) degree from University of Agriculture Faisalabad. His research areas are Green cloud computing, Wireless Body Area Network and Adhoc Networks. 